\begin{tabular}{lllllllllllllllllllllllllllllllll}
\hline$R$ & $E$ & $V$ & I & S & T & A & D & E & E & S & T & U & D & I & O & S & I & N & T & E & R & N & A & C & I & O & N & A & L & E & S
\end{tabular}

\title{
La política de la transferencia internacional de tecnología en América Latina
}

Óscar Plaza

Este trabajo es un estudio de las políticas de transferencia internacional de tecnología en los países de América Latina. Pretendemos mostrar la necesidad de tener a los nuevos desarrollos tecnológicos y los cambios de políticas económicas como marco de referencia para entender la evolución del pensamiento acerca de la transferencia internacional de tecnología en la región y el consecuente diseño y rediseño de políticas al respecto. Como corolario, en primer lugar, intentamos adelantar la tesis de que, paradójicamente, estas políticas han sido simultáneamente exitosas y frustrantes. En segundo lugar, en términos de tecnología política, la transferencia internacional de tecnología juega un rol importante en la estabilidad social del futuro.

Para los efectos de nuestro estudio, por un lado, examinamos la evolución histórica del pensamiento y políticas de transferencia internacional de tecnología en América Latina a fin de entender por qué se le ha considerado importante. Examinamos también el diseño, implementación y resultados institucionales de las políticas respectivas. Por otro lado, en un intento de contribuir con algunos alcances teóricos, organizamos este trabajo proponiendo una diferenciación de etapas históricas en la evolución del pensamiento y políticas acerca de la transferencia internacional de tecnología en América Latina. Este esfuerzo teórico trata de reafirmar la idea de que los cambios tecnológicos y las modificaciones de las políticas económicas forzaron drásticas transformaciones en las políticas de transferencia internacional de tecnología en la región, afectando a su vez radicalmente el rol y función de las instituciones y actores involucrados. 
LA ERA DEL DESARROLLO NACIONAL

\section{a) Perspectiva histórica}

espués de la Segunda Guerra Mundial, dado el poder demostrado por las nuevas tecnologías en el campo de batalla, se produjo un consenso internacional acerca de la necesidad de promover el desarrollo científico y tecnológico al servicio de la paz. Durante los años cincuenta, conceptos como desarrollo, industrialización, planificación, organización, e identidad de la "comunidad científica nacional" tomaron mucha fuerza dentro de los círculos de opinión intelectual de los países latinoamericanos. Esta actitud era consecuente, por lo demás, con la institucionalidad internacional surgida después de la guerra, la cual propició explícitamente esta línea de conceptos.

\section{El modelo de política de desarrollo científico-tecnológico de la Unesco}

apuntaba a hacer visible
la investigación científica
nacional de alto nivel.

La Unesco fundó un Departamento Científico y, desde éste, comenzó a propugnar un modelo de política de desarrollo científico/tecnológico. El objetivo declarado del modelo era hacer posible la "investigación científica nacional" de alto nivel. La teoría sustentadora del modelo era que el conocimiento científico y la experiencia en investigación científica iban a "empujar" el desarrollo socioeconómico nacional. La premisa básica de esta línea de pensamiento era que "buena ciencia" -física, química, y otras disciplinas básicas- crearía "buena tecnología", definida esta última como la aplicación del conocimiento científico a la procura de metas de productividad económica.

En 1970, la Asamblea General de las Naciones Unidas aprobó la Estrategia Internacional para el Desarrollo, 1971-1980 (UN 1971a). Esta estrategia consideraba explícitamente la contribución de la ciencia y la tecnología para el desarrollo de los países en vías de desarrollo y la importancia de la transferencia internacional de tecnología (UN 1971b). En este contexto, el Consejo Económico y Social de las Naciones Unidas instruyó a sus Comisiones Regionales que tomaran iniciativas concretas en este ámbito. Fue así como la Comisión Económica para América Latina y el Caribe (CEPAL) decidió promover la transferencia de tecnología en la región, para lo cual estudió y recomendó la creación de sistemas de información científica y tecnológica, de patentes y de transferencia tecnológica entre los países del área. Adicionalmente, hubo varios esfuerzos sobre la misma materia dentro de América Latina.

No es de sorprender, entonces, que ya en la década de los sesenta el programa estadounidense Alianza para el Progreso en América Latina hiciera del desarrollo científico y tecnológico un objetivo explícito de dicho programa. En línea con el espíritu "desarrollista" de la Alianza, la Organización de los Estados Americanos (OEA) creó el Departamento de Asuntos Científicos y Tecnológicos. Acto se- 
guido, este Departamento puso en marcha un programa destinado a iniciar un proceso de desarrollo científico y tecnológico en la región.

Este conjunto de circunstancias políticas internacionales creó las condiciones para lanzar un ambicioso plan de transferencia internacional de tecnología hacia América Latina y dentro de ella. Hasta hoy, el esfuerzo de aquella época constituye el más completo y coordinado programa con este fin realizado en la región. Fue así como de acuerdo con el Programa Regional de Desarrollo Científico y Tecnológico de la OEA, la División de Planes y Estudios de su Departamento de Asuntos Científicos y Tecnológicos preparó un marco conceptual para definir y ejecutar un "proyecto piloto" de transferencia internacional de tecnología en América Latina.

\section{b) El Proyecto Piloto de Transferen- cia de Tecnología}

El Proyecto Piloto de Transferencia de Tecnología de la OEA (OEA, 1972) ejemplifica la preeminencia del enfoque de desarrollo económico nacional y la influencia de factores y actores internacionales externos en el pensamiento y las consiguientes políticas de ciencia y tecnología en los países latinoamericanos. En efecto, durante los años '60, la Organización para la Cooperación y el Desarrollo Económico (OECD) creó un programa para enfatizar y propiciar el rol de la ciencia y la tecnología en el desarrollo.

Este programa propone expresamente "un método para definir los requerimientos de investigación científica y tec- nológica en relación al desarrollo económico". (OCDE, 1968a:19) Este método consiste en crear "grupos de trabajo" para ejecutar "proyectos pilotos". El objetivo explícito de estos proyectos piloto era construir instituciones capaces de administrar eficientemente políticas de ciencia y tecnología, pues ellas son "de particular necesidad en países en vías de desarrollo". (OCDE, 1968b:147).

\section{El programa de la ocDE planteaba una correlación básica entre desarrollo tecnológico y desarrollo económico.}

El esquema conceptual de la OCDE planteaba, en primer lugar, una correlación básica entre desarrollo tecnológico y desarrollo económico. En segundo lugar, la falta de desarrollo tecnológico se debería a la inexistencia de un mercado tecnológico nacional. De acuerdo con este enfoque, la falta de demanda tecnológica interna por parte del sector productivo nacional tiene como contrapartida la importación de tecnología foránea, la cual, a su vez, sería parte del mercado internacional de tecnología. Por esta razón, las políticas nacionales de ciencia y tecnología imperiosamente tienen que considerar el proceso internacional de transferencia de tecnología (Gonod 1972). Este proceso determinaría la distribución internacional de tecnología y funcionaría de acuerdo con principios básicos de mercado. En consecuencia, la inversión extranjera, los joint-ventures, los contratos de 
compra de tecnología (licencias, patentes, marcas), y las transacciones de materias primas, de bienes de capital y de soporte técnico serían actos propios del mercado internacional de transferencia de tecnologías. Acciones que involucraran bienes no transables, tales como el libre flujo de conocimiento científico y la asistencia técnica y profesional, no formarían parte del mercado internacional de tecnología, pero deberían ser consideradas en las políticas de transferencia internacional de tecnología.

De esta manera, el enfoque y metodología propuesto por la OCDE, fuertemente influenciados por el pensamiento francés en materia de política de ciencia y tecnología, fue llevado a la práctica por la OEA en el Proyecto Piloto de Transferencia de Tecnología, impulsado por el gran interés estadounidense de llevar a cabo un programa tecnológico para el desarrollo (OEA, 1971 y 1972b). El resultado más importante del proyecto fue la creación de grupos de "expertos en políticas de tecnología" en cada uno de los países participantes en el proyecto. Estos expertos se transformaron en los promotores políticos y administradores de las políticas de tecnología en los países latinoamericanos. Es de notar que la gran mayoría de estos nuevos expertos no provenía de la comunidad científica, sino más bien eran especialistas provenientes de la economía, la administración pública y la ingeniería. Precisamente, estos profesionales se transformaron en expertos en política de ciencia y tecnología debido a su participación en el proyecto piloto de la OEA. Las comunidades científicas de los países de la región llegaron a ser importantes grupos de presión en apoyo de las políticas propuestas y ejecutadas por los expertos, pero muchos científicos eran fuertes opositores a los criterios técnicoeconómicos de los expertos. Las comunidades científicas querían investigación científica, en circunstancias que los expertos querían cambio tecnológico susceptible de medir en términos de desarrollo económico.

\section{El Proyecto Piloto de la OEA contribuyó a la consolidación institucional de las políticas científicas y tecnológicas de la región.}

Otro importante resultado del Proyecto Piloto fue la institucionalización de las políticas científicas y tecnológicas en América Latina. Por supuesto, este proyecto no es el único factor, pero sin duda contribuyó de manera significativa a la consolidación institucional de dichas políticas. Hasta entonces, en sus discursos, los políticos latinoamericanos se referían con frecuencia al importante papel de la ciencia y la tecnología para el desarrollo, pero había una notoria falta de institucionalidad encargada de diseñar y aplicar políticas al respecto. En gran parte y gracias al Proyecto Piloto, la política de ciencia y tecnología logró un lugar dentro del aparato burocrático del Estado en América Latina.

En términos de la transferencia de tecnología propiamente tal, los resultados del Proyecto Piloto fueron ambiguos. Sin lu- 
gar a dudas, el proyecto amplió significativamente el conocimiento del proceso de transferencia internacional de ciencia y de tecnología y la capacidad de administración del mismo en Latinoamérica. No obstante, el mejor conocimiento del proceso y la exitosa institucionalización de las políticas no significaron una evidente mejora del estatus internacional de los sistema científico-tecnológicos nacionales, ni tampoco una clara contribución al desarrollo socioeconómico nacional en la región.

\section{c) El paradigma}

El Proyecto Piloto de Transferencia de Tecnología no fue sólo un programa para mejorar el proceso de transferencia internacional de tecnología dentro de América Latina y hacia la región, sino que constituyó además un intento deliberado de establecer un procedimiento para originar, institucionalizar y administrar políticas públicas en una nueva área de interés político. La meta era comenzar a operar, tan pronto fuera posible, como un sector institucionalizado de la administración del Estado. El resultado fue muy similar al de la creación de nuevos organismos públicos mediante un proceso formal de debates y decisiones por parte de las instancias políticas, pero la singularidad de la experiencia residió en el énfasis casi exclusivo en una secuencia de decisiones de carácter fundamentalmente administrativo acerca de aspectos de gestión de los asuntos científicos y tecnológicos. Nunca se abordó directamente la política científica y tecnológica como materia de na- turaleza política que la comunidad nacional debía debatir y los órganos políticos del Estado decidir. Todo esto en un contexto en que la ideología consensual de la época enfatizaba y priorizaba el "desarrollo nacional", daba a la "ciencia y tecnología" un rol importante en ese propósito y consideraba al Estado como el actor central y principal.

\section{El proyecto piloto consideraba aspectos relacionados con la ingeniería, lo administrativo y lo político.}

El concepto de proyecto piloto para el desarrollo de la ciencia y la tecnología utilizó un método de acción que consideraba simultáneamente tres aspectos: el de la ingeniería, el administrativo y el político. Desde el punto de vista del primero, la idea era desarrollar un prototipo que creara grupos de acción, pequeños y cohesionados, con la misión específica de promocionar la institucionalización de la política científica y tecnológica en América Latina. El aspecto administrativo aspiraba a proporcionar un mecanismo de coordinación tanto a nivel nacional como regional. La acción paralela de varios grupos actuando con el mismo ideario, los mismos objetivos y un mismo plan en distintos países de la región se consideraba indispensable para el éxito de dicha empresa. Desde el ángulo político, la idea era la factibilidad del proyecto. El objetivo no era lograr un triunfo ideológico, sino la capacidad de alinear tras el pro- 
yecto a actores y eventos políticos muy disímiles. En definitiva, la idea del proyecto piloto fue probar un modelo para enfrentar el desafío administrativo que presentaba un propósito político en una nueva área de las políticas públicas. Dado el contexto ideológico de la época, muy favorable a una estrategia del desarrollo nacional encabezada por el Estado, el modelo fue exitoso en términos de institucionalizar la política científica y tecnológica y la transferencia internacional de tecnología como parte de dicha política.

\section{LA ERA DE LA TRANSICIÓN}

A mediados de la década de los ochenta, estaba claro que, no obstante el éxito de la institucionalización de la política de transferencia internacional de tecnología, los resultados obtenidos eran pobres en cuanto a generación de capacidad tecnológica nacional, de influencia en al aparato productivo y en definitiva, de impacto en el desarrollo socio económico nacional. A nivel internacional se estaban produciendo dos fenómenos de profunda influencia en el pensamiento y acción en el ámbito tecnológico. El primero tenía que ver con la tecnología misma. El nuevo campo de la así llamada "tecnología avanzada" prácticamente terminó con la fase de desarrollo tecnológico de la era industrial, en circunstancias en que, hasta entonces, el pensamiento y la política científico-tecnológicos de la región habían tenido a la industrialización como marco de referencia. En segundo lugar, el pensa- miento y la acción en materia de política económica se estaba revirtiendo dramáticamente. De un enfoque de desarrollo "hacia adentro" mediante la "sustitución de importaciones" se pasaba a un desarrollo "hacia afuera" mediante el "mercado abierto" en materia de comercio exterior. La administración institucional del flujo de transferencia internacional de tecnología era perfectamente complementaria con un mercado internacional cerrado, pero difícil de conciliar con una política de libre mercado.

\section{La "tecnología avanzada" terminó con la fase de desarrollo tecnológico de la era industrial.}

En esta coyuntura, tres enfoques acerca de la transferencia internacional de tecnología se disputaban el interés de los círculos académicos y los centros de decisión políticos. Un primer enfoque, el de la "escuela de los incentivos de precio", enfatizaba la idea del "precio correcto", Los seguidores de esta escuela creían que los precios de mercado, que reflejan los costos sociales, no sólo guiarán la selección de la tecnología más eficiente, sino que crearán los incentivos para el desarrollo de las tecnologías más apropiadas para el desarrollo (Teece 1981). No es necesario enfatizar que este pensamiento se enmarca perfectamente en políticas económicas que propugnan la privatización, la liberalización y los mercados abiertos al comercio internacional.

El enfoque opuesto sostuvo que la promoción de la innovación tecnológica 
no puede dejarse al mecanismo de precios. Lo que se necesitaría es una decisión premeditada de invertir en desarrollo tecnológico y controlar la transferencia internacional de tecnología. Los principales sostenedores de este enfoque fueron los expertos formados en la era de la ciencia y tecnología para el desarrollo nacional. Estos expertos constituyeron la espina dorsal de la infraestructura institucional creada en aquella era. En su mérito, cabe acotar que dichos especialistas han sido también capaces de mantener en el tiempo esa institucionalización, si bien actualmente ella no tiene mayor relevancia en el aparato burocrático del Estado, ni tampoco en el debate político nacional.

La "escuela de la reforma radical" adoptó un enfoque más amplio. Arguyó que tanto los productos como los métodos de producción son elementos inherentes al sistema socioeconómico. Por lo tanto, sería imposible que países avanzados crearan la "tecnología apropiada" para países en vía de desarrollo, tanto en términos de productos como de factores de producción. No obstante, esta escuela no desarrolló un modelo práctico que permitiera diseñar políticas nacionales que abordaran los desafíos tecnológicos de los países y regiones menos desarrolladas, en particular en América Latina. En aquel entonces fue un novedoso concepto de "tecnologías alternativas", en la actualidad sólo sirve de inspiración a proyectos tecnológicos atractivos, pero puntuales. Su mayor contribución fue que creó una nueva actitud, esto es, una conducta de pensamiento crítico para estudiar cualquier propuesta tecnológica.

\section{El concepto de "tecnologías alternativas" creó una actitud crítica para estudiar cualquier propuesta tecnológica.}

En el ámbito más contingente, también se asistía a una fuerte tensión entre la perspectiva con que se miraba al proceso de transferencia internacional de tecnología desde las empresas multinacionales y los centros intelectuales y políticos de los países latinoamericanos (Teece 1976). Las primeras miraban su inversión en tecnología como un proceso continuo, que debe producir retornos financieros tal como debe hacerlo cualquier inversión (Ethier and Markusen, 1996). En cambio, a las autoridades de los países latinoamericanos les preocupaba más que nada el acceso a tecnologías ya existentes en países desarrollados. Desde esta óptica, tendían a ver la inversión en tecnología por parte de empresas multinacionales como costos del pasado ("costos hundidos") que no requieren reembolso (Pack and Saggi, 1997).

Dada esta diferencia de percepciones, no es de extrañar que los organismos políticos latinoamericanos hayan intentado controlar el flujo internacional de tecnología. Es así como se inició una era de elaboradas legislaciones destinadas a asegurar que las tecnologías provenientes de empresas multinacionales beneficiaran al 
sector productivo privado y público nacional y al país receptor en general. Sin embargo, desde un comienzo causó inquietud que la nueva legislación pudiera no sólo desincentivar la transferencia de tecnología, sino también no aliviar en nada los supuestos abusos de las empresas multinacionales. En rigor, en la práctica, la legislación demostró ser muy ambiciosa, complicada y no operativa en un contexto internacional de rápido cambio tecnológico. Mas la transferencia internacional de tecnología continuó, a veces, restringida por la legislación; a veces, traspasándola.

El sello de la discusión de este período de transición estuvo dado por las implicaciones que tenía para el desarrollo tecnológico la reestructuración de tendencias en la esfera mundial. El desarrollo de las "tecnologías avanzadas" y la formación de estructuras económicas y financieras globales estaban desafiando el concepto mismo de políticas tecnológicas, especialmente en lo que respecta a la naturaleza "nacional" contenida intrínsecamente en el concepto. Una de las formas de la reestructuración de la economía mundial fue la emergencia de alianzas entre compañías financieras y de creación de tecnologías con estructuras de producción y comercialización que, sobrepasando el concepto de varios mercados nacionales de acción, ven al mundo entero como su mercado natural. El resultado de este proceso fue la alta especialización tecnológica de grandes empresas y la rápida evolución de las tecnologías que crean. Es entonces que se dieron las condiciones para una verdadera economía "multinacional". En este nuevo escenario, incluso las economías de países más avanzados comenzaron a evolucionar dentro de "estructuras de dependencia" global. Por supuesto, las "estructuras de dependencia" funcionan de manera "asimétrica", por lo tanto, las economías de países avanzados gozan de una autonomía relativa mayor, pero aún más importante, son más gravitantes en la determinación de estas estructuras que las economías de países en vías de desarrollo. No obstante, la mera factibilidad de una economía nacional autónoma comenzó a ser altamente improbable.

\section{La alta especialización tecnológica de grandes empresas dio lugar a que surgiera una verdadera economía multinacional.}

En este nuevo contexto, la idea del control del proceso de transferencia internacional de tecnología como instrumento de la política tecnológica nacional colapsó. Si algo se podía dar por cierto es que las nuevas tecnologías no reconocen nacionalidad y menos la de un país en vías de desarrollo, por lo que el diseño de esquemas de control y regulación de la distribución internacional de tecnología empezó a mostrar signos de futilidad. Comenzó entonces el cuestionamiento del concepto mismo de ciencia y tecnología para el desarrollo y de transferencia internacional de tecnología como uno de los instrumentos para administrar el cambio tecnológico en dichos países. 


\section{LA ERA DE LA ANSIEDAD}

La transferencia de tecnología desde países desarrollados hacia países en vías de desarrollo ha continuado y crecido en años recientes. Por un lado, se arguye que la perspectiva de mejoramiento del nivel económico de los países en vías de desarrollo depende en gran medida de la incorporación y adaptación de tecnologías ya existentes en países avanzados. Por otro lado, existe amplio consenso en que la tecnología transferida no siempre se adecua a las necesidades ni a los recursos de estos últimos países. También existe una generalizada percepción de que la brecha tecnológica entre estos dos grupos de países se acrecienta, si no a nivel de elites, a nivel de la población en su conjunto (Spencer 1970, Glass and Kamal 1998).

\section{Hay amplio consenso en que la tecnología transferida a los países en desarrollo no siempre se adecua a sus necesidades y recursos.}

No es difícil entender el gran interés que existe hoy en América Latina respecto de la transferencia internacional de tecnología. En primer lugar, se teme que el rápido ritmo de cambio tecnológico de los países desarrollados acreciente la brecha tecnológica de la región. También existe amplia evidencia de que las nuevas tecnologías avanzadas se transfieren cada vez más rápidamente de una industria a otra, con lo cual ningún sector de la economía y de la sociedad está libre de sus efectos. Además, las nuevas tecnologías se difunden a ritmo creciente de una nación a otra, a través de todo el orbe. Pero lo más importante es que se comprueba la existencia de una percepción generalizada en la inteligencia contemporánea de que estamos en medio de una revolución tecnológica. Esta revolución estaría transformando la naturaleza misma de la actividad económica, y con ello la infraestructura material de la sociedad, tal como el vapor, la electricidad y el motor de combustión interna lo hicieron durante la revolución industrial. La actual revolución tecnológica tendría sus comienzos en la electrónica, mostrando hoy todo su potencial en el campo de la informática. También asistimos a los comienzos del desarrollo de la biotecnología iniciado con el descubrimiento del ADN en los años cincuenta y que en la actualidad empieza a tener efectos directos en áreas tan sensibles como alimentos y medicina. Agréganse a esto tecnologías potencialmente revolucionarias en el área de los materiales y la fotónica.

Es así como la situación actual se caracteriza por el continuo cambio tecnológico en los países avanzados y la rápida difusión de la nueva tecnología a nivel global, incluyendo sectores de elite de países en vías de desarrollo (Arnold, Guy, Hanna 1995). Pero este desarrollo tecnológico a nivel mundial no alivia el atraso, la marginalidad y el consiguiente sufrimiento en los países en vías de desarrollo. Más aún, pareciera que la transferencia internacional de tecnología hacia estos países tensiona su tejido social y su 
identidad cultural. La raíz del conflicto que genera el cambio tecnológico se debe a la naturaleza dual de toda nueva tecnología; por un lado, conlleva el surgimiento de nuevos valores, por ejemplo, eficiencia, instantaneidad y conexión global; por el otro, destruye antiguos valores, a saber, artesanía, contemplación y lealtad local. Las sociedades avanzadas tienen una cultura tecnológica con más tradición y son las iniciadoras de la innovación tecnológica, lo cual les permite sobreponerse con más facilidad a la destrucción de valores tradicionales. En los países en vías de desarrollo, la nueva tecnología trae consigo el reemplazo de sus propios valores por aquellos imbuidos en tecnologías foráneas y sin que el acervo de una tradición de cultura tecnológica medie la transición. En estos países, la transferencia internacional de tecnología resulta así subversiva.

\section{El aporte de una fuerza de trabajo tecnológicamente capacitada se ha convertido en principal factor de producción de las empresas multinacionales.}

La infraestructura material de la llamada "nueva economía" tiene su base en la "tecnología avanzada" y en el liderazgo de las empresas multinacionales que han desarrollado modelos comerciales para estas tecnologías. Para seguir siendo competitivas estas nuevas empresas dependen en alto grado de la innovación tecnológica. En consecuencia, el conocimiento que aporta una fuerza de trabajo tecnológicamente capacitada se ha convertido en el principal factor de producción de estas empresas. Siendo así, la existencia de una fuerza de trabajo barata, con escasos conocimientos tecnológicos, sería un elemento que desincentivaría la inversión en los países en vías de desarrollo. Sólo cuando una unidad territorial, sea una economía nacional o local, pueda producir y reproducir una fuerza de trabajo tecnológicamente capacitada, estas empresas estarían dispuestas a moverse desde regímenes de ventajas absolutas a otros de ventajas comparativas en términos de creación de valor agregado. Parecería entonces que estaríamos frente a una de requisitos básicos para que un país partícipe en la nueva economía.

Sin embargo, la capacidad de producir y reproducir fuerza de trabajo tecnológicamente capacitada es solamente un factor necesario, pero no suficiente, para incorporar a un país al camino del desarrollo tecnológico y económico contemporáneo. Filipinas, por ejemplo, tiene un sistema de educación terciaria relativamente avanzado, pero ha sido incapaz de capitalizar este recurso humano. Su sector económico de tecnología avanzada permanece atrapado en un régimen de creación de valor agregado de ventajas absolutas, debido a que internacionalmente, dispone de relativa abundancia de mano de obra barata. Es así como, a pesar de la transferencia internacional de tecnología, muchos países en vías de desarrollo permanecen atrapados en su condición de economías de alto coeficiente de mano de obra barata y de bajo valor 
agregado dentro de la división internacional de la fuerza de trabajo. Es en estos países, más que en otros, donde la nueva economía, basada en tecnologías avanzadas y en su consiguiente división tecnológica polarizante, ha contribuido en mayor medida a la masiva desigualdad social. Por sí, la nueva tecnología no crea las condiciones para el desarrollo ni mejora las condiciones de vida de la mayoría de la población de estos países. La transferencia internacional de tecnología no es, entonces, una respuesta cabal a los problemas del desarrollo de los países atrasados.

He aquí entonces el dilema que presenta la transferencia internacional de tecnología a los países latinoamericanos y en vías de desarrollo en general. Por un lado, se entienden la tensión y la subversión que esta nueva tecnología trae consigo, pero al mismo tiempo, se comprende que esta tecnología es el factor clave de poder en la nueva economía global. Peor, la tecnología avanzada encierra la promesa de desarrollo económico sin necesidad de utilizar mano de obra y recursos naturales abundantes y baratos, que constituyen las tradicionales ventajas absolutas de los países en vías de desarro1lo. La verdadera brecha internacional parecería estar definiéndose, no tanto en términos de países diferentemente afectados por las nuevas tecnologías, sino más bien entre los países integrados a la nueva estructura económica internacional y los excluidos de ella. Esto, porque no tienen la capacidad mínima para incorporar las nuevas tecnologías, necesarias para competir en una economía globalizada donde el costo de la mano de obra y los recursos naturales no importa mayormente (Castells 1994).

\section{Al parecer, la brecha internacional se define entre los países integrados a la nueva estructura económica internacional y los excluidos de ella.}

En otras palabras, el mundo avanzado puede seguir su desarrollo sin necesidad del resto. La tecnología avanzada encierra la posibilidad de tornar redundantes a los países en vías de desarrollo. Percibiendo esta posibilidad, las elites de estos Estados tratan desesperadamente de traer tecnología avanzada mediante el proceso de transferencia internacional de tecnología. El acceso a nueva tecnología les permite sentirse parte del mundo contemporáneo, aun cuando no sea el caso para aumentar el bienestar de su población. Por el contrario, la mayor parte de la gente de los países en vías de desarrollo siente que el nuevo mundo tecnologizado ni siquiera los explota, de acuerdo con el significado del término en economía política y como sucedió en la era de la industrialización, sino simplemente los ignora. Siendo así, la tecnología política nos diría que el futuro se definirá en la medida en que la gran mayoría de esta población, que se siente abandonada, crea que puede ponerse al día con el proceso de tecnologización, o bien, en que pierda toda esperanza y, entonces, no faltarán quienes piensen que simplemente es mejor arrasar con todo el sistema socioeconómico contem- 
poráneo, pues frente a la desesperanza todo costo es inconmensurable.

\section{La transferencia internacional de tecnología es un poderoso instrumento de control social.}

Gracias al cambio tecnológico, los países incrementan su capacidad de crear riqueza y adquieren los medios para alcanzar objetivos sociales. Éste es el punto de vista positivo del "enfoque tecnológico" del desarrollo y la transferencia internacional de tecnología. Sin embargo, esta última es también un poderoso instrumento de control social. Aquellos con capacidad de generar y administrar tecnología, como es el caso de las empresas multinacionales, o de usar tecnología, como es la situación de quienes pueden adquirirla en países en vía de desarrollo, tienen el poder de definir problemas, delimitar alternativas de solución, e influir en la toma de decisiones políticas. Éste es el punto de vista negativo del "enfoque social" del desarrollo tecnológico y la transferencia internacional de tecnología. Sin embargo, la evolución de la tecnología muestra que el control de ésta, junto al poder relativo que confiere, es inherentemente inestables, y se disipan más rápidamente que el poder basado en mecanismos tradicionales de control político. De ahí que en términos de tecnología política, el verdadero interés esté centrado en la participación del proceso de desarrollo tecnológico, esto es, invención, innovación y desarrollo de tecnologías y no en el control de una tecnología en particular.

\section{Conclusiones}

La conclusión de estas reflexiones destaca que la política de transferencia internacional de tecnología en Latinoamérica se desdibujó totalmente desde el paso de una era que llamaríamos de la "tecnología para el desarrollo" a nuestra actual "globalización tecnológica". Se partió de una era de pensamiento asertivo, en que se creyó conocer el camino al desarrollo y en el cual la tecnología era una variable independiente capaz de jugar un rol de palanca para el desarrollo nacional. Sin embargo, llegamos a comienzos del siglo XXI a una época de ansiedad, donde la tecnología es condición y consecuencia de la globalización. Dada esta condición, la tecnología actúa como una variable sistémica que se resiste a una clara conceptualización, sea teórica u operacional.

En el contexto de las Américas, fue posible pensar en proyectos internacionales de cooperación tecnológica con características de "verticalidad" debido a la existencia de países en diferentes estadios de desarrollo tecnológico. Esto significaba que, a través de la participación de países avanzados -los Estados Unidos y Canadá- y países en vías de desarrollo -naciones latinoamericanas-, era posible originar un proceso fluido de transferencia internacional de tecnología. Sin embargo, ninguno de los países de América Latina tenía los recursos, la organización, la capacidad administrativa y la 
tradición tecnológica como para transformar la transferencia de conocimiento científico y tecnológico en "impulsor" del desarrollo socioeconómico. Si bien fue posible institucionalizar la política científica y tecnológica y la administración de la transferencia internacional de tecnología, no se tuvo éxito en términos de crear un sistema científico tecnológico "nacional e independiente" que proveyera de nueva tecnología al sector productivo. En mi opinión, los exiguos resultados se debieron no sólo a fallas de concepción y ejecución de las políticas, sino también a debilidades en el pensamiento acerca de ciencia y tecnología. Esto indicaría la necesidad de repensar el fenómeno tecnológico contemporáneo y su impacto en América Latina.

Nuestra premisa es que la transferencia internacional de tecnología en América Latina continuará, cualquiera sea la modalidad de administración, pública o de mercado, dado que hoy en día la tecnología es un factor crítico en la economía y la sociedad en su conjunto. Los problemas asociados a este proceso de transferencia son de naturaleza múltiple, desde la naturaleza misma de la tecnología "apropiada" a las variadas formas de negociación de términos y condiciones de las formas de transferencia. La transferencia internacional de tecnología puede ser un poderoso instrumento para el desarrollo de la región, pero al mismo tiempo un vehículo de destrucción del tejido social y de la identidad cultural. Como sea, la difusión internacional de tecnología es fuente potencial de trastornos sociales nacionales e internacionales.
En términos de política, el dilema para los países latinoamericanos es que prescindir de la transferencia internacional de tecnología en los términos y modalidades en que se da en la economía global no es ni siquiera una alternativa. Durante la era del "desarrollo nacional", estos países hicieron un esfuerzo deliberado y serio para controlar y administrar, vía instituciones de Estado, la transferencia internacional de tecnología. La idea era "traer" el proceso de desarrollo tecnológico al país de manera de crear un "sistema de ciencia y tecnología" nacional que generara tecnología para el sistema productivo nacional. La "mentalidad tecnológica" iba a generar no sólo desarrollo económico, sino que también, modernizar la sociedad. Después de una era fundacional de las políticas de ciencia y tecnología todo el esquema conceptual e institucional se paraliza. Entra en crisis la "mentalidad industrializadora" en que se basaban las políticas debido al nuevo contexto de una economía mundial "global" que tenía su fundamento en tecnologías avanzadas, empresas multinacionales que desarrollan modelos comerciales para ellas y políticas económicas de mercado abierto en materia de comercio exterior. Entonces, los países latinoamericanos -en vías de desarrollo- generalmente se ven ante la disyuntiva de lanzarse a un sistema global basado en procesos tecnológicos de los cuales son receptores pasivos, o simplemente ser marginados. En términos de tecnología política, pareciera que esta última alternativa es más temida porque actualmente la transferencia internacional de tecnología es un esfuerzo desesperado por "ser parte de". 
Los potenciales beneficios de la transferencia internacional de tecnología en países en vías de desarrollo podrían llegar a manifestarse si a nivel internacional se diera una coordinación de estrategias de desarrollo, políticas tecnológicas y opciones valóricas concordantes. La discusión internacional debe también considerar una mayor desagregación entre los países en vías de desarrollo. La competencia tecnológica de estos países difiere mucho. Además, las tecnologías mismas son muy diferentes, no sólo en aspectos técnicos, sino también en el tipo de demanda organizacional y administrativa. Por lo tanto, el ser competente no se da en términos de la tecnología en general, sino en términos de especialización, lo cual puede abrir ventanas de oportunidad a muchos de estos países. La única ventaja comparativa que puede tener un país en términos de tecnología política internacional, es la capacidad de adaptarse y administrar el cambio tecnológico mediante ajustes sociales flexibles. Desafortunadamente, esto es más fácil para los países en vía de desarrollo, pues se enfrentan a una peligrosa conjunción de dramático cambio tecnológico y poca o nula experiencia en el arte de administrar dicho cambio.

\section{REFERENCIAS BIBLIOGRÁFICAS}

Amadeo, Eduardo (1979). "National Science and Technology Council in Latin America: Achievement and Failures of the First Ten Years" en Integration of Science and Technology with Development, Miguel Wionczek y Thomas D. Babatunde, editores. New York, Pergamon Press, págs. 146-166

Arnold, Erik, Ken Guy y Nagy K. Hanna (1995). The Diffusion of Information Technology: Experiences of Industrial Countries and Lessons for Developing Countries, World Bank Discussion Paper \# 281.

Campo, Alvaro (1993). "Contexto de Ciencia y Tecnología en Latinoamérica con énfasis en los países miembros del Convenio Andrés Bello”, en La Cooperación Multilateral en Ciencia y Tecnología, y los Sistemas de Ciencia y Tecnología de los Países del Convenio Andrés Bello, Alvaro Campo Cabal y Henry Yesid Bernal, editores, Santafé de Bogotá, D.C., Colombia

Castells, Manuel y Peter Hall (1994). Technopoles of the World: The Making of Twenty First Century Industrial Complexes, New York, Routhledge.

Congress of the United States, Office of Technology Assessment (1984). Technology Transfer to the Middle East, Report, Washington, D.C.

Davenport, Thomas H. y Laurence Prusack (2000). Working knowledge: How Organizations Manage What they Know, Boston, MA, Harvard Business School Press

Ethier, Wilfred J. y James R. Markusen (1996). "Multinational Firms, Technology Diffusion and Trade", Journal of International Economics, 62, págs. 1-16

Glass, Amy J. y Kamal Saggi (1998). "International Technology Transfer and the Technology Gap", Journal of Development Economics, 55, 369-398.

Gonod, Pierre (1972). Diagnóstico General Sobre la Transferencia Tecnológica en América Latina. Washington, D.C., OEA, Departamento de Asuntos Científicos. 
Huenemann, Ralph (1984). China's Open Door Policy: The Quest for Foreign Technology and Capital, a study of China's Special Trade, Vancouver, Canadá, University of British Columbia Press.

I.D.R.C. (1976). Science and Technology Policy Implementation in Less-Developed Countries: Methodological Guidelines for the STPI project, Otawa, Canada, International Development Research Centre (IDRC).

Lavados, Jaime (1979). "Organization of Scientific and Technological Development in Latin America”, en Integration of Science and Technology..., op. cit., pág. 139-148.

Mokyr, Joel (1999). The Lever of Riches. Technological Creativity and Economic Progress, New York, Oxford University Press.

N.U. (1971a). Programa de Acción de la Asamblea General para el Segundo Decenio de las Naciones Unidas para el Desarrollo, New York, N.U.

N.U. (1971b). La Gran Experiencia: La Ciencia y la Tecnología en el Segundo Decenio para el Desarrollo, by Robin Clarke, New York, Centro de Información Económica y Social.

Pack, Howard and Kamal Saggi (1997). "Inflows of Foreign Technology and Indigenous Technological Development", Review of Development Economics, 1, págs. 81-98

O.A.S (1971). Las Funciones y Objetivos de la Experiencia Piloto Sobre Transferencia y Desarrollo Técnico, Washington D.C., O.E.A., Departamento de Asuntos Científicos.

O.A.S. (1972a). General Description of the O.A.S. Pilot Project for Transfer of Technology, General Secretariat of the O.A.S., Washington D.C., Department of Scientific Affairs.

O.A.S. (1972b). Reunión del Grupo de Trabajo sobre el Proyecto Piloto de Transferencia de Tecnología, Washington D.C., O.E.A, Departamento de Asuntos Científicos.

O.A.S. (1975). Informe Final: Proyecto Piloto de Transferencia de Tecnología, Washington D.C., O.E.A., Departamento de Asuntos Científicos.

O.E.C.D. (1968a). Science Policy and Development. A Synthesis of the Methods of Analyses used in the Pilot Teams Projects, Paris, France, OECD, DAS/SPR/68.28.

O.E.C.D. (1968b). L'Experience des Equipes Pilotes, Conference d'Evaluation, Science et Development, Introduction: Historique et Objectifs, Paris, France, Organisation de Cooperation et de Development Economiques, págs. 7-14.

Ralph, Eric, Greg Felker, Henry Ergas and Melvin Goldman (1997). Technology Institutions and Policies: Their Role in Developing Technological Capabilities in Industry, World Bank Technical Paper \# 383.

RAND Corporation (1998). Monitoring and Controlling the International Transfer of Technology. Published by RAND.

Rosenberg, Nathan y Claudio Frischtak, editores (1985). International Technology Transfer: concepts, measures and comparisons, New York, Praeger.

Rosenberg, Nathan (1994). Exploring the Black Box: Technology, Economics, and History, New York: Cambridge University Press.

Schmookler, Jacob C. (1966). Invention and Economic Growth, Cambridge, Massachusetts, Harvard University Press.

Spencer, Daniel (1970). Technology Gap in Perspective. Strategy of International Technology Transfer, New York, Spartan Books.

Teece, David (1981). "The Market for Know-How and the Efficient International Transfer of Technology", en Technology Transfer: New Issues, New Analyses, Alan W. Heston and Howard Pack, eds., The Annals of the American Academy of Political and Social Science, págs. 81-96

Wionczek, Miguel (1979). "Science and Technology Planning in LDCs", en Integration of Science and Technology..., op. cit., pág. 167-177. 